\title{
Percutaneous Access of Nondilated Renal Collecting Systems
}

\author{
Charles E. Ray Jr., MD, PhD, FSIR ${ }^{1} \quad$ Anthony C. Brown, MD ${ }^{1} \quad$ Mitchell T. Smith, MD, MS ${ }^{1}$ \\ Paul J. Rochon, MD, RPVI ${ }^{1}$ \\ ${ }^{1}$ Department of Radiology, School of Medicine, University of \\ Colorado, Aurora, Colorado \\ Semin Intervent Radiol 2014;31:98-100 \\ Address for correspondence Charles E. Ray Jr, MD, PhD, FSIR, \\ Department of Radiology, School of Medicine, University of Colorado, \\ 12401 E. 17th Avenue, Room 526, Aurora, CO 80045 \\ (e-mail: Charles.ray@ucdenver.edu).
}

Interventional radiology (IR) has seen an increase in the numbers of patients referred for urinary diversion in patients with ureteral leaks. This increase has corresponded to the increasing use of complicated surgical procedures for lower urinary tract tumors. These procedures are often relatively difficult to perform for two reasons: first, the renal collecting systems are typically completely decompressed due to the leak; second, many of these patients have recently undergone a significant operation via a ventral approach, making patient positioning difficult. The latter issue is relatively easy to control with the aid of anesthesiologists and the creative use of bolsters and pillows, although in the worst case scenario patients may have to be positioned in a lateral decubitus position (further complicating an already difficult procedure). The discussion below outlines the authors' procedural algorithm in gaining percutaneous collecting system access in this difficult patient population.

\section{Technique}

1. Standard ultrasound-guided access. Although the collecting system may be completely decompressed, many of these patients will have chronically dilated renal collecting systems due to prior ureteral obstruction. If the obstruction was present for many months, the renal collecting system will typically remain dilated for many weeks, months, or years even after relief of the obstruction; indeed, in many chronically obstructed patients, the renal collecting system never returns to a normal caliber. In addition, in some patients (particularly those who are thin or those with chronic medical renal disease, where the collecting system is relatively easy to distinguish from the remainder of the kidney), these physiologic effects can be taken advantage of when placing percutaneous nephrostomy tubes. The authors will use ultrasound (US) for any initial attempt given its low risk and ready accessibility.
2. Administration of intravenous contrast to opacify the renal collecting system for fluoroscopically guided calyceal access. In patients with normal renal function, the intravenous (IV) administration of approximately 50 to $80 \mathrm{~mL}$ of iodinated contrast can be helpful to visualize the renal collecting system. Some authors have advocated the use of IV furosemide and a $250 \mathrm{~mL}$ normal saline bolus to facilitate temporary dilation of nondilated calyces (see Bibliography). If adequately visualized, an attempt can be made to directly puncture one of the posterior renal calyces in anticipation of definitive nephrostomy tube placement. The operator must remain cognizant of the fact that administered contrast will typically drain very quickly from the kidney and out of the rent in the lower



Figure 1 CT scan of a patient with hydronephrosis in the prone position. This image demonstrates the en face orientation of the posterior calyx when visualized from a straight posteroanterior approach (open arrow); the anterior calyx (solid arrow) is seen longitudinally when viewed from the same approach. This differentiation is very helpful when fluoroscopically determining which complex represents the posterior calyceal complex, which is typically the preferred calyx to percutaneously access. CT, computed tomography.
Issue Theme Renal Malignancies; Guest Editors, Bradley B. Pua, MD and David C. Madoff, MD, FSIR
Copyright @ 2014 by Thieme Medical Publishers, Inc., 333 Seventh Avenue, New York, NY 10001, USA. Tel: +1(212) 584-4662.
DOI http://dx.doi.org/ 10.1055/s-0033-1363849. ISSN 0739-9529. 


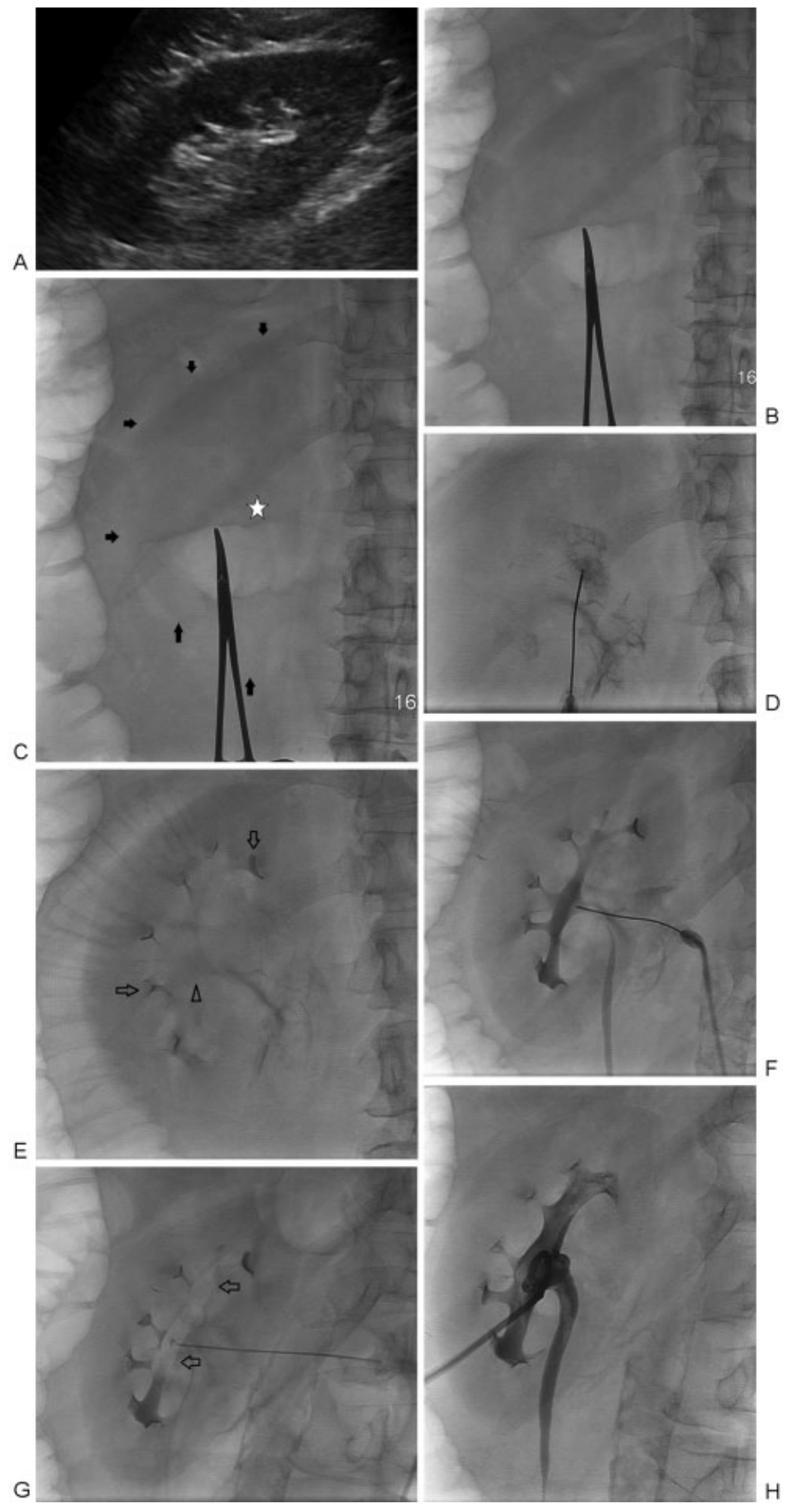

Figure 2 Percutaneous nephrostomy tube placement in a patient with decompressed calyces. (A) Ultrasound demonstrates normal caliber of the calyces without evidence for hydronephrosis. (B) Spot fluoroscopic image demonstrating the faint renal outline. The overlying hemostat is lateral to the anticipated location of the renal pelvis. (C) Same image as B, with arrowheads outlining the renal shadow and the anticipated location of the renal pelvis (star). (D) Extracalyceal contrast following attempt at fluoroscopically assisted renal pelvis needle placement for a two-stick technique. The renal pelvis could not be successfully accessed using fluoroscopic-guided technique. (E) Following the administration of $80 \mathrm{~mL}$ iodinated contrast IV, the renal pelvocalyceal system is faintly visualized. (Arrows-renal calyces; arrowhead-renal pelvis.) (F) Using contrast in the renal pelvis as a fluoroscopic guide, a 21-gauge needle has been placed into the renal pelvis for a two-stick technique. Contrast injected through the needle now fills the entire pelvocalyceal collecting system. (G) Room air $(10 \mathrm{~mL})$ was injected via the renal pelvis needle to differentiate the anterior and posterior calyces. Air floats to the nondependent posterior calyces (arrows) in this prone patient. (H) Using the air in the posterior calyces as a target, a second needle was used to access the posterior calyx for definitive nephrostomy tube placement. 
urinary tract. Although this is not dangerous to the patient, it allows the operator very little time (often just a couple of minutes) to obtain fluoroscopically guided access. In addition, it can sometimes be difficult to differentiate the anterior from the posterior calyces, particularly in patients with malrotation of their kidneys. One helpful hint is that in using a straight posteroanterior approach, the posterior calyces (the calyces that should be targeted during percutaneous access) are typically seen en face, whereas the anterior calyces are visualized longitudinally (-Fig. 1). A direct "down-the-barrel" puncture of a posterior calyx can be attempted; however, rarely is this approach used for actual tube placement as this would result in eventual placement of the catheter in a less than optimal location for patient comfort (straight out the back rather than the flank). Given the short time frame of calyceal opacification following IV contrast administration, and difficulty in distinguishing anterior versus posterior calyces, the authors tend to use IV contrast administration in conjunction with the two-stick technique described below.

3. Two-stick technique. The two-stick technique can be very helpful during difficult access procedures; however, with the ubiquitous use of US in most IR laboratories today, the two-stick technique is rarely used (and may be infrequently taught). In this technique, the first needle is placed directly into the renal pelvis from a straight posteroanterior approach. This can be performed with US in some cases, although fluoroscopic guidance is more typically used. Fluoroscopic guidance can be based on osseous landmarks (typically approximately $2 \mathrm{~cm}$ lateral to the transverse process of the L2 vertebral body); however, with improvements in fluoroscopy, the renal shadow can often be seen directly, facilitating placement of the needle into the anticipated region of the renal pelvis. In addition, prior cross-sectional imaging, particularly computed tomographic or magnetic resonance images, should be reviewed as these may provide the operator with anatomic landmarks from which to estimate the location of the renal pelvis.

A 21-gauge needle is used for the initial renal pelvis puncture to minimize complications from inadvertent puncture of the renal hilar vessels (which probably happens with some regularity). If no urine is aspirated after the first needle pass, the approach angle is altered by approximately 10 degrees in one direction or another; choice of the altered angle is operator dependent and somewhat random. Once access is obtained in the renal pelvis, contrast $(10 \mathrm{~mL})$ is injected to confirm adequate placement of the renal pelvis needle. The authors will typically use half-strength contrast to facilitate visualization of the wire that will eventually be placed via the second needle. Carbon dioxide or room air (the authors use the latter) is then injected while directly watching under fluoroscopy. Gas will rise to the nondependent calyces, in which a prone patient is the posterior calyceal complex; this represents the target calyx for puncture. If the air is injected without watching under fluoroscopy, the injected air will simulate and be indistinguishable from bowel gas. Once the appropriate posterior calyx is identified, an appropriate skin site for exit of the nephrostomy tube can be chosen. The authors typically choose an exit site, a hands breadth medial to the posterior axillary line. A hemostat is placed at the skin exit site; angling the X-ray tube in both the mediolateral and craniocaudal projection allows superimposition of the hemostat and the targeted calyx, facilitating a "down the barrel" approach for the catheter placement. Once the calyx is accessed, standard technique is performed for final tube placement.

4. Combined procedure with retrograde ureterography. If none of the above procedures work, a combined approach with urology may be most appropriate. By performing a combined approach, contrast can be injected retrograde into the ureter of interest through a cystoscope, providing a direct fluoroscopic target of the calyces for either a singleor double-stick technique. This approach also allows distension of the renal calyces if enough contrast is injected. In some circumstances, such as patients with neobladders or loop ileostomies, urologists may find it very difficult or even impossible to access the new ureteral anastomosis, in which case a combined procedure will not prove helpful. In any case, due to the logistics (and cost) of such an approach, most IR physicians use this as a last-ditch approach.

-Fig. 2 represents images from a patient in which nearly all of the above techniques were used. As is commonly the case, many patients will require such a multifaceted approach.

Accessing decompressed calyces is one of the more difficult procedures for an interventionalist to perform. Add the relatively high risk of such procedures (due to multiple punctures in a very vascular organ) to the technical difficulty, and one can easily understand the need to optimize both the patient and technical aspect of the procedure before proceeding.

\section{Bibliography}

Dagli M, Ramchandani P. Percutaneous nephrostomy: technical aspects and indications. Semin Intervent Radiol 2011;28(4): 424-437

Patel U, Hussain FF. Percutaneous nephrostomy of nondilated renal collecting systems with fluoroscopic guidance: technique and results. Radiology 2004;233(1):226-233

Yagci C, Ustuner E, Atman ED, Baltaci S, Uzun C, Akyar S. Diuretic agent and normal saline infusion technique for ultrasound-guided percutaneous nephrostomies in nondilated pelvicaliceal systems. Cardiovasc Intervent Radiol 2013;36(2):492-497 